\title{
Visualizing the long non-coding subgenomic flavivirus RNAs in solution
}

Yupeng Zhang ${ }^{1, \boldsymbol{\oplus}}$, Yikan Zhang ${ }^{1, \uparrow}$, Junfeng Ma ${ }^{1}$, Xianyang Fang ${ }^{1 *}$

1 Beijing Advanced Innovation Center for Structural Biology, School of Life Sciences, Tsinghua University, Beijing 100084, China. E-mail: fangxy@tsinghua.edu.cn

Most mosquito-borne flaviviruses such as Zika virus (ZIKV), Dengue virus (DENV) and West Nile virus (WNV) produce an abundance of long non-coding subgenomic RNAs (known as sfRNAs) in infected cells that link to viral pathogenicity and immune evasion. Until now, structural characterization of these sfRNAs remains limited. In combination with small angle x-ray scattering (SAXS) and computational modeling, we studied the tertiary structures of individual and combined subdomains and visualized the accessible 3D conformational spaces of complete sfRNAs from DENV2, ZIKV and WNV, respectively, in solution. The individual xrRNA1s and xrRNA2s adopt similar structures in solution as the crystal structure of ZIKV xrRNA1, the xrRNA12s form compact structures with reduced flexibility. While the DB12 of DENV2 is extended, the DB12s of ZIKV and WNV are compact due to the formation of intertwined double pseudoknots in proximity. All 3'SLs share similar rod-like structures. The complete sfRNAs are extended and sample a large conformational space in solution, which may enable their roles in modulating several cellular pathways by interacting with diverse viral and host proteins. Our work also highlights the combination of SAXS and computational modeling as ideal tools for exploring the accessible 3D conformational spaces of other lncRNAs in solution. 